DFTT 31/00

arXiv:hep-ph/0007155

\title{
A frequentist analysis of solar neutrino data
}

\author{
M.V. Garzelli and C. Giunti \\ INFN, Sez. di Torino, and Dip. di Fisica Teorica, Univ. di Torino, I-10125 Torino, Italy
}

(July 13, 2000)

\begin{abstract}
We calculate with Monte Carlo the goodness of fit and the confidence level of the standard allowed regions for the neutrino oscillation parameters obtained from the fit of solar neutrino data. We show that the values of the goodness of fit and of the confidence level of the allowed regions are significantly smaller than the standard ones. Using Neyman's method, we also calculate exact allowed regions with correct frequentist coverage. We show that the standard allowed region around the global minimum of the least-squares function is a reasonable approximation of the exact one, whereas the size of the other regions is dramatically underestimated in the standard method.
\end{abstract}

PACS numbers: 26.65.+t, 14.60.Pq, 14.60.Lm 


\section{INTRODUCTION}

The standard method to analyze solar neutrino data in terms of neutrino oscillations consists in performing a least-squares fit. However, for the reasons described in Section II the standard least-squares analysis of solar neutrino data is approximate from a statistical point of view.

In this paper we present statistical methods based on Monte Carlo numerical calculations that allow to improve the implementation of the least-squares fit of solar neutrino data. In Section [1] we review the standard method and we discuss why its approximate assumptions could lead to significant inaccuracy in the results. In Section [II we present a Monte Carlo method that allows to calculate the goodness of fit of solar neutrino data. In Section IV we present a Monte Carlo method that allows to calculate the confidence level of the usual allowed regions in the space of the neutrino oscillation parameters. In Section $\square$ we present an implementation to solar neutrino analysis of the classical frequentist Neyman method that allows to calculate exact confidence regions with correct coverage.

Since the purpose of this paper is to illustrate different methods for the statistical analysis of solar neutrino data, we consider for simplicity only the data relative to the total rates measured in the Homestake [1] and Super-Kamiokande [2] experiments, and the weighted average of the total rates measured in the two Gallium experiments GALLEX [3] and SAGE [4]. The values of these rates are given in Table I of Ref. [5]. Updated results of the SuperKamiokande experiment and first results of the new GNO experiment have been presented in the recent Neutrino 2000 conference [6]. Since the numerical calculations presented here take a long time and were started before the Neutrino 2000 conference, we do not take into account the new data. A complete analysis including the new data and the SuperKamiokande data relative to the electron energy spectrum and the zenith-angle distribution is under way and will be published elsewhere []].

Neutrino oscillations depend on the mass-squared difference $\Delta m^{2} \equiv m_{2}^{2}-m_{1}^{2}$ and on the mixing angle $\vartheta$, that is restricted in the interval $[0, \pi / 2]$. Traditionally solar neutrino data have been analyzed in terms of the parameters $\Delta m^{2}$ and $\sin ^{2} 2 \vartheta$, that determine the probability of neutrino oscillations in vacuum. However, it has recently been shown that the parameter $\tan ^{2} \vartheta$ is more convenient for finding the allowed regions in the interval $\pi / 4 \leq \vartheta \leq$ $\pi / 2$ when matter effects are important [10,11]2. Moreover, the parameter $\tan ^{2} \vartheta$ allows a better view of the regions at large mixing angles with respect to the usual parameter $\sin ^{2} 2 \vartheta$. Hence, in the following we analyze the solar neutrino data in terms of the parameters $\Delta m^{2}$ and $\tan ^{2} \vartheta$.

Our calculation of the theoretical event rates follows the standard method described in

${ }^{1}$ Here we consider the minimal two-neutrino model, although more complicated models are possible (see [8,9]).

${ }^{2}$ For the same reason the parameter $\tan ^{2} \vartheta$ has been employed in the framework of three-neutrino mixing 12 14 and the parameter $\sin ^{2} \vartheta$ has been employed in the framework of four-neutrino mixing [15]. 
several papers for matter-enhanced MSW [16 transitions 17 19 and vacuum oscillations 20,19. We calculate the MSW survival probability of $\nu_{e}$ 's in the Sun using the standard analytic prescription [21, 18, 17,9] and the level-crossing probability appropriate for an exponential density profile [22,17]. We calculate the regeneration in the Earth using a two-step model of the Earth density profile [23 27], that is known to produce results that do not differ appreciably from those obtained with the correct density profile. We have used the tables of neutrino fluxes, solar density and radiochemical detector cross sections available in Bahcall's web page [28]. For simplicity we have neglected the matter effects that slightly affect the vacuum oscillation solutions of the solar neutrino problem, as discussed in [29,30].

\section{STANDARD STATISTICAL ANALYSIS}

The traditional way to find the values of the neutrino oscillation parameters $\Delta m^{2}, \tan ^{2} \vartheta$ allowed by solar neutrino data is to perform a least-squares fit, often called " $\chi^{2}$ fit". In this method the estimates $\widehat{\Delta m^{2}}, \widehat{\tan ^{2} \vartheta}$ of the parameters $\Delta m^{2}, \tan ^{2} \vartheta$ are obtained by minimizing the least-squares function

$$
X^{2}=\sum_{j_{1}, j_{2}}\left(R_{j_{1}}^{(\mathrm{thr})}-R_{j_{1}}^{(\mathrm{exp})}\right)\left(V^{-1}\right)_{j_{1} j_{2}}\left(R_{j_{2}}^{(\mathrm{thr})}-R_{j_{2}}^{(\mathrm{exp})}\right)
$$

where $V$ is the covariance matrix of experimental and theoretical uncertainties, $R_{j}^{(\exp )}$ is the

event rate measured in the $j^{\text {th }}$ experiment and $R_{j}^{(\text {thr })}$ is the corresponding theoretical event rate, that depends on $\Delta m^{2}$ and $\tan ^{2} \vartheta$.

The standard method for the calculation of the covariance matrix $V$ is the one presented in Refs. 31,32, in which the independent uncertainties $\sigma_{j}^{2}$ of the experimental rates $R_{j}^{(\exp )}$, and the uncertainties of the theoretical rates $R_{j}^{(\mathrm{thr})}$ are added in quadrature. Here we use this method, with the only difference that we assume a complete correlation of the errors of the averaged cross sections for the fluxes in each experiment [33]. Since these correlations are not known, the choice of complete correlations is the safest approach. Hence, using the notation of Refs. [31, 32] the covariance matrix $V$ is given by

$$
V_{j_{1}, j_{2}}=\delta_{j_{1}, j_{2}} \sigma_{j_{1}}^{2}+\delta_{j_{1}, j_{2}}\left(\sum_{i_{1}} R_{i_{1} j_{1}}^{(\mathrm{thr})} \Delta \ln C_{i_{1} j_{1}}^{(\mathrm{thr})}\right)^{2}+\sum_{i_{1}, i_{2}} R_{i_{1} j_{1}}^{(\mathrm{thr})} R_{i_{2} j_{2}}^{(\mathrm{thr})} \sum_{k} \alpha_{i_{1} k} \alpha_{i_{2} k}\left(\Delta \ln X_{k}\right)^{2}
$$

where

3 The indices $j, j_{1}, j_{2}=1,2,3$ indicate the three solar neutrino experiments GALLEX+SAGE [3, [4], Homestake [1] and Super-Kamiokande [2], respectively. The indices $i, i_{1}, i_{2}=1, \ldots, 8$ denote the solar neutrino fluxes produced in the eight solar thermonuclear reactions $p p$, pep, Нep, Be, B, $\mathrm{N}, \mathrm{O}, \mathrm{F}$, respectively. The index $k=1, \ldots, 11$ indicate the eleven input astrophysical parameters in the SSM (see Refs. 31, 32]). 


$$
R_{i j}^{(\mathrm{thr})}=\phi_{i}^{\mathrm{SSM}} C_{i j}^{(\mathrm{thr})}
$$

is the event rate in the $j^{\text {th }}$ experiment due to the neutrino flux $\phi_{i}^{\text {SSM }}$ produced in the $i^{\text {th }}$ thermonuclear reaction in the sun according to the SSM and $C_{i j}^{(\text {thr })}$ is the corresponding energy-averaged cross section that depends on $\Delta m^{2}$ and $\tan ^{2} \vartheta$. The quantity $\Delta \ln C_{i j}^{(\text {thr })}=$ $\Delta C_{i j}^{(\mathrm{thr})} / C_{i j}^{(\mathrm{thr})}$ is the relative uncertainty of the energy-averaged cross section $C_{i j}^{(\mathrm{thr})}$, that is taken to be approximately equal to the one calculated without neutrino oscillations.

The quantities $X_{k}$ are the input astrophysical parameters in the SSM, whose relative uncertainties $\Delta \ln X_{k}$ determine the correlated uncertainties of the neutrino fluxes $\phi_{i}^{\mathrm{SSM}}$ through the logarithmic derivatives

$$
\alpha_{i k}=\frac{\partial \ln \phi_{i}^{\mathrm{SSM}}}{\partial \ln X_{k}} .
$$

The values of $\Delta \ln C_{i j}^{(\mathrm{thr})}, \alpha_{i k}, \Delta \ln X_{k}$ are given in Ref. 32.

Notice that, since the theoretical rates $R_{i j}^{(\mathrm{thr})}$ depend on $\Delta m^{2}$ and $\tan ^{2} \vartheta$, also the covariance matrix $V$ depends on $\Delta m^{2}$ and $\tan ^{2} \vartheta$.

In the traditional method the minimum $X_{\min }^{2}$ of (1) provides the estimate of the neutrino oscillation parameters, usually called "best-fit values", $\widehat{\Delta m^{2}}$ and $\widehat{\tan ^{2} \vartheta}$. The goodness of the fit is estimated by calculating the probability to observe a minimum of $X^{2}$ larger than the one actually observed assuming for $X_{\min }^{2}$ a $\chi^{2}$ distribution with $N_{\text {exp }}-N_{\text {par }}=1$ degrees of freedom, where $N_{\exp }=3$ is the number of experimental data points (the sums over $j_{1}$ and $j_{2}$ in Eq. (11) are from 1 to $N_{\text {exp }}$ ) and $N_{\text {par }}=2$ is the number of fitted parameters. Calling $\alpha$ this probability, one says that the fit is acceptable at $100 \alpha \%$ CL. If $\alpha$ is larger than a minimum acceptable value, usually $\sim 10^{-2}$, the fit is considered to be acceptable and one can proceed further to determine the uncertainties in the determination of the parameters $\Delta m^{2}$ and $\tan ^{2} \vartheta$ (the allowed regions in parameter space).

The standard regions of the parameters allowed at $100 \beta \%$ CL are those that satisfy the condition

$$
X^{2}=X_{\min }^{2}+\Delta X^{2}(\beta),
$$

where $\Delta X^{2}(\beta)$ is given by the value of $\chi^{2}$ such that the cumulative $\chi^{2}$ distribution for $N_{\text {par }}=2$ degrees of freedom (the number of parameters) is equal to $\beta$. Common values for $\beta$ are $0.90(1.64 \sigma), 0.95(1.96 \sigma), 0.99(2.58 \sigma), 0.9973(3.00 \sigma)$, which give $\Delta X^{2}(0.90)=4.61$, $\Delta X^{2}(0.95)=5.99, \Delta X^{2}(0.99)=9.21, \Delta X^{2}(0.9973)=11.83$.

This procedure would be correct if the theoretical rates $R_{j}^{(\mathrm{thr})}$ depended linearly on the parameters $\Delta m^{2}$ and $\tan ^{2} \vartheta$ to be determined in the fit and the errors $R_{j}^{(\text {thr })}-R_{j}^{(\text {exp })}$ were multinormally distributed with constant covariance matrix $V$. Indeed, if these requirements were realized one could prove that $X^{2}$ has a $\chi^{2}$ distribution with $N_{\exp }=3$ degrees of freedom, $X_{\text {min }}^{2}$ has a $\chi^{2}$ distribution with $N_{\exp }-N_{\text {par }}=1$ degrees of freedom, and $X^{2}-X_{\min }^{2}$ has a $\chi^{2}$ distribution with $N_{\text {par }}=2$ degrees of freedom (see [34 36]). In this case the $X^{2}$ function would depend quadratically on the parameters and there would be only one allowed region with ellipsoidal form in the space of the parameters $\Delta m^{2}$ and $\tan ^{2} \vartheta$. 
In the case of solar neutrino data the gaussian distribution of experimental and theoretical uncertainties seems to be widely accepted, although it is not clear if this assumption is appropriate for the theoretical errors. On the other hand it is clear that

1. The theoretical rates $R_{j}^{(\mathrm{thr})}$ do not depend at all linearly on the parameters $\Delta m^{2}$, $\tan ^{2} \vartheta$. This is the reason why there are several allowed regions in the $\tan ^{2} \vartheta-\Delta m^{2}$ plane (or the more traditional $\sin ^{2} 2 \vartheta-\Delta m^{2}$ plane) and these regions do not have elliptic form (see [37,5).

2. The covariance matrix $V$ is not constant, but depends on $\Delta m^{2}$ and $\tan ^{2} \vartheta$, as remarked after Eq. (州).

3. The errors $R_{j}^{(\mathrm{thr})}-R_{j}^{(\exp )}$ are not multinormally distributed, because although the fluxes $\phi_{i}^{\text {SSM }}$ and the cross sections $C_{i j}^{(\text {thr })}$ are assumed to be multinormally distributed, their products (3), that determine the theoretical rates through the relations

$$
R_{j}^{(\mathrm{thr})}=\sum_{i} R_{i j}^{(\mathrm{thr})}
$$

are not multinormally distributed (see [38]).

Hence, the usual method of calculating the goodness of fit and the allowed regions in the $\tan ^{2} \vartheta-\Delta m^{2}$ plane is not guaranteed to give correct results, i.e. the goodness of fit could be significantly different from $100 \alpha \%$ and the confidence level of the regions enclosed by borders with constant $X^{2}=X_{\min }^{2}+\Delta X^{2}(\beta)$ could be significantly different from $100 \beta \%$.

We believe that the largest correction is due to the non-linear dependence of the theoretical rates $R_{j}^{\text {(thr) }}$ from the parameters $\Delta m^{2}, \tan ^{2} \vartheta$, that causes the existence of more than one local minima of the least-squares function $X^{2}$. This implies that there are more possibilities to obtain good fits of the data and the true goodness of fit is likely to be smaller than $100 \alpha \%$. Also, in repeated experiments the global minimum has significant chances to occur far from the true (unknown) value of the parameters $\Delta m^{2}, \tan ^{2} \vartheta$, with a smaller probability that the allowed regions cover the true value with respect to the linear case. Hence, we expect that the true confidence level of a usual 100 $\beta \%$ CL allowed region is smaller than $\beta$.

In the following sections of this paper we perform a least-squares fit of the solar neutrino data using the $X_{\min }^{2}$ estimator for the neutrino oscillation parameters $\Delta m^{2}, \tan ^{2} \vartheta$. We assume the usual gaussian distribution for the experimental and theoretical uncertainties. In Section [II] we calculate the goodness of fit using the Monte Carlo method, that is applicable in any case in which the distribution of the uncertainties is known (see, for example, Section 15.6 of [36]). In Section IV we calculate with the Monte Carlo method the confidence level of the usual allowed regions in the $\tan ^{2} \vartheta-\Delta m^{2}$ parameter space. In Section $\nabla$ we implement the classical frequentist Neyman method for finding exact confidence regions with correct coverage at a given confidence level.

\section{GOODNESS OF FIT}

In order to calculate the goodness of fit, our method proceeds as follows (see, for example, Section 15.6 of [36]). We estimate the best-fit values of $\Delta m^{2}, \tan ^{2} \vartheta$ through the minimum 
of $X^{2}$ in Eq. (11) and we call these best-fit values $\widehat{\Delta m^{2}}, \widehat{\tan ^{2} \vartheta}$. Then we assume that $\widehat{\Delta m^{2}}$, $\widehat{\tan ^{2} \vartheta}$ are reasonable surrogates of the true values $\Delta m_{\text {true }}^{2}, \tan ^{2} \vartheta_{\text {true }}$ and the probability distribution of the differences $\widehat{\Delta m^{2}}(k)-\widehat{\Delta m^{2}}, \widehat{\tan }^{2} \vartheta_{(k)}-\widehat{\tan ^{2} \vartheta}$ is not too different from the true distribution of the differences $\widehat{\Delta m^{2}}(k)-\Delta m_{\text {true }}^{2}, \widehat{\tan ^{2} \vartheta_{(k)}}-\tan ^{2} \vartheta_{\text {true }}$ in a large set of best-fit parameters ${\widehat{\Delta m^{2}}}_{(k)},{\widehat{\tan ^{2}}}_{(k)}(k=1,2, \ldots)$ obtained with hypothetical experiments.

Using $\widehat{\Delta m^{2}}, \widehat{\tan ^{2} \vartheta}$ as surrogates of the true values, we generate $N_{s}$ synthetic random data sets with the usual gaussian distribution for the experimental and theoretical uncertainties. We apply the least-squares method to each synthetic data set, leading to an ensemble of simulated best-fit parameters ${\widehat{\Delta m^{2}}}_{(s)},{\widehat{\tan ^{2} \vartheta_{(s)}}}$ with $s=1, \ldots, N_{s}$, each one with his associated $\left(X_{\min }^{2}\right)_{s}$. Then we calculate the goodness of the fit as the fraction of simulated $\left(X_{\min }^{2}\right)_{s}$ in the ensemble that are larger than the one actually observed, $X_{\min }^{2}$.

We calculate the synthetic data sets generating random neutrino fluxes $\phi_{i}$ with a multinormal distribution centered on the SSM fluxes $\phi_{i}^{\mathrm{SSM}}$ and having the covariance matrix

$$
V_{i_{1}, i_{2}}^{(\phi)}=\phi_{i_{1}}^{\mathrm{SSM}} \phi_{i_{2}}^{\mathrm{SSM}} \sum_{k} \alpha_{i_{1} k} \alpha_{i_{2} k}\left(\Delta \ln X_{k}\right)^{2}
$$

We also generate random energy-averaged cross sections $C_{i j}$ with a multinormal distribution centered on the theoretical energy-averaged cross sections $C_{i j}^{(\mathrm{thr})}$ corresponding to $\widehat{\Delta m^{2}}$, $\widehat{\tan ^{2} \vartheta}$ and having the completely correlated covariance matrix for each independent experiment $j$

$$
V_{i_{1}, i_{2}}^{(j)}=C_{i_{1} j} \Delta \ln C_{i_{1} j} C_{i_{2} j} \Delta \ln C_{i_{2} j} .
$$

Then, we calculate the rates $R_{j}=\sum_{i} \phi_{i} C_{i j}$. Finally, we generate random synthetic experimental rates $R_{j}^{(s)}$ with normal distribution centered on $R_{j}$ and standard deviation equal to that of the actual experimental data $\left(\sigma_{j}\right)$. The synthetic experimental rates are inserted in the least-squares function (1) in place of $R_{j}^{(\exp )}$ in order to find the minimum $\left(X_{\min }^{2}\right)_{s}$ and its associated best-fit parameters ${\widehat{\Delta m^{2}}}_{(s)},{\widehat{\tan ^{2} \vartheta}}_{(s)}$.

The results of our calculations are reported in Table [. The global minimum of the leastsquares function (11), $X_{\min }^{2}=0.42$, occurs in the SMA regionf for $\Delta m^{2}=5.1 \times 10^{-6} \mathrm{eV}^{2}$ and $\tan ^{2} \vartheta=1.6 \times 10^{-3}$. The results reported in the "SMA" row of Table $\mathbb{1}$ have been obtained taking $\widehat{\Delta m^{2}}=5.1 \times 10^{-6} \mathrm{eV}^{2}$ and $\widehat{\tan ^{2} \vartheta}=1.6 \times 10^{-3}$. We first restricted the allowed region of the mixing parameters around the SMA region $\left(10^{-4} \leq \tan ^{2} \vartheta \leq 3 \times 10^{-2}\right.$ and $3 \times 10^{-7} \mathrm{eV}^{2} \leq \Delta m^{2} \leq 10^{-4} \mathrm{eV}^{2}$ ) and obtained the local value of the goodness of fit, reported in the "local" column of Table [1. This value is almost equal (even slightly larger) to the standard one obtained assuming a $\chi^{2}$ distribution with one degree of freedom, reported in the "standard GOF" column of Table II. Hence, we conclude that locally the usual method to evaluate the goodness of fit is reliable.

${ }^{4}$ Here we use the standard terminology for the allowed regions (see [37,5]): SMA for $\Delta m^{2} \sim$ $5 \times 10^{-6} \mathrm{eV}^{2}, \tan ^{2} \vartheta \sim 10^{-3}$, LMA for $\Delta m^{2} \sim 3 \times 10^{-5} \mathrm{eV}^{2}, \tan ^{2} \vartheta \sim 0.3, \mathrm{LOW}$ for $\Delta m^{2} \sim 10^{-7} \mathrm{eV}^{2}$, $\tan ^{2} \vartheta \sim 0.5$, VO for $\Delta m^{2} \lesssim 10^{-8} \mathrm{eV}^{2}$. 


\begin{tabular}{|c|l|c|c|c|c|}
\hline \multicolumn{2}{|c|}{ region } & \multicolumn{3}{|c|}{ Goodness of fit } \\
\cline { 3 - 6 } SMA & $\begin{array}{l}X_{\min }^{2}=0.42 \\
m^{2}=5.1 \times 10^{-6} \mathrm{eV}^{2} \\
\tan ^{2} \vartheta=1.6 \times 10^{-3}\end{array}$ & $51.8 \%$ & $53.7 \%$ & $48.4 \%$ & $39.6 \%$ \\
\hline LMA & $\begin{array}{l}X_{\min }^{2}=3.46 \\
\Delta m^{2}=1.5 \times 10^{-5} \mathrm{eV}^{2} \\
\tan ^{2} \vartheta=0.30\end{array}$ & $6.3 \%$ & $6.1 \%$ & & \\
\hline LOW & $\begin{array}{l}X_{\min }^{2}=6.53 \\
\Delta m^{2}=1.3 \times 10^{-7} \mathrm{eV}^{2}\end{array}$ & $1.1 \%$ & $1.9 \%$ & & \\
\hline \multirow{2}{*}{ VO } & $\begin{array}{l}X_{\min }^{2} \vartheta=0.55 \\
\Delta m^{2}=9.4 \times 10^{-11} \mathrm{eV}^{2} \\
\tan ^{2} \vartheta=0.38\end{array}$ & $25.6 \%$ & $14.2 \%$ & & \\
\hline
\end{tabular}

TABLE I. Goodness of fit of solar neutrino data calculated with more than one million synthetic data sets. The first two columns indicate in which region the surrogate of the true values of the neutrino oscillation parameters has been assumed to be, the corresponding values of $X_{\min }^{2}$ and the values of the surrogates. The third column indicates the goodness of fit calculated with the standard method, i.e. assuming a $\chi^{2}$ distribution with one degree of freedom. The fourth column reports the goodness of fit calculated locally, i.e. restricting the allowed values of the parameters around the region in which the assumed surrogates of the true values lie. The fifth column reports the goodness of fit calculated restricting the allowed values of the parameters to the MSW region (9). The sixth column reports the goodness of fit calculated without any restriction on the allowed values of the parameters.

However, when we extend the allowed region of the mixing parameters to all the MSW region

$$
10^{-4} \leq \tan ^{2} \vartheta \leq 2, \quad 10^{-8} \mathrm{eV}^{2} \leq \Delta m^{2} \leq 10^{-3} \mathrm{eV}^{2} \quad \text { (MSW region) }
$$

and when we add also the VO region

$$
0.1 \leq \tan ^{2} \vartheta \leq 1, \quad 10^{-11} \mathrm{eV}^{2} \leq \Delta m^{2} \leq 10^{-8} \mathrm{eV}^{2} \quad \text { (VO region) }
$$

we obtain the values reported, respectively, in the "MSW" and "global" columns of Table I, which are significantly smaller than the one obtained with the standard method. As remarked in Section II, this is due to the non-linear dependence of the theoretical rates from the neutrino oscillation parameters, that implies that there are more possibilities to obtain good fits of the data with respect to the linear case. Therefore, we conclude that the standard method, although valid locally (when the allowed region of the parameters is restricted around the SMA region the linear assumption is approximately correct), is not valid in general and should not be trusted if there is more than one allowed region.

In order to check the local validity of the standard method we have also assumed that $\widehat{\Delta m^{2}}$ and $\widehat{\tan ^{2} \vartheta}$ have the values corresponding to the local minima of $X^{2}$ in the LMA, LOW 
and VO regions, restricting the allowed values of the parameters around the corresponding regions. The results are reported in the "LMA", "LOW" and "VO" rows of Table $\mathbb{1}$. One can see that the standard method is locally acceptable for the LMA and LOW solutions, but it largely overestimates the goodness of fit in the case of the VO solution. This is due to the fact that the theoretical rates are highly non-linear functions of the neutrino oscillation parameters in the VO region (10), resulting in several disjointed allowed regions.

The "MSW" and "global" entries in the "LMA", "LOW" and "VO" rows of Table If are empty because it is meaningless to calculate the goodness of fit allowing values of the parameters in which the fit is better than the one in the assumed surrogate of the true values of the parameters.

Summarizing the results of this section, we have shown that if there were only one allowed region in the space of the neutrino oscillation parameters, or if there are valid reasons to restrict the allowed region of the parameters around one of the SMA, LMA, LOW solutions, the standard method to calculate the goodness of fit is approximately reliable. On the other hand, if there are more than one allowed regions, the standard method to calculate the goodness of fit is not reliable and the goodness of fit must be calculated numerically, with Monte Carlo, as we have done. This happens if one considers the MSW region (9) of the neutrino oscillation parameters, which contains three allowed regions (SMA, LMA and LOW), or the VO region (10), that contains several allowed regions, or all the parameter space $(\mathrm{MSW}+\mathrm{VO})$.

\section{CONFIDENCE LEVEL OF ALLOWED REGIONS}

In order to calculate the confidence level of the allowed regions it is necessary first to understand what is its meaning. The $100 \beta \%$ CL allowed regions are defined by the property that they belong to a set of allowed regions obtained with hypothetical experiments and the regions belonging to this set cover (i.e. include) the true value of the parameters with probability $\beta$.

Given the usual " $100 \beta \%$ CL" allowed regions in the space of the neutrino oscillation parameters we can calculate their confidence level $\beta_{\mathrm{MC}}$ with a method similar to the one

described in the previous section for the goodness of fit. We assume that $\widehat{\Delta m^{2}}, \widehat{\tan ^{2} \vartheta}$ are reasonable surrogates of the true values $\Delta m_{\text {true }}^{2}, \tan ^{2} \vartheta_{\text {true }}$ and we generate a large number of synthetic data sets. We apply the standard procedure to each synthetic data set and obtain the corresponding " $100 \beta \%$ CL" allowed regions in the space of the neutrino oscillation parameters. Then we count the number of synthetic " $100 \beta \%$ CL" allowed regions that cover

the assumed surrogate $\widehat{\Delta m^{2}}, \widehat{\tan ^{2} \vartheta}$ of the true values. The ratio of this number and the total number of synthetically generated data set gives the confidence level $\beta_{\mathrm{MC}}$ of the " $100 \beta \%$ CL" allowed regions.

The results of our calculations are reported in Table [1]. As we have done in the previous section for the goodness of fit, we calculated first the local confidence levels restricting the allowed values of the parameters around the region whose local minimum of $X^{2}$ gives the assumed surrogates of the true values ("local" column of Table II). Then we calculated the confidence levels restricting the allowed values of the parameters to the MSW region (9) assuming the surrogates of the true values in the local minima of $X^{2}$ of the SMA, LMA 


\begin{tabular}{|c|c|c|c|c|}
\hline \multirow{2}{*}{ region } & \multicolumn{4}{|c|}{ Confidence level of allowed regions } \\
\cline { 2 - 5 } & standard CL & local & MSW & global \\
\hline \multirow{4}{*}{ SMA } & $90.00 \%(1.64 \sigma)$ & $90.11 \%(1.65 \sigma)$ & $87.22 \%(1.52 \sigma)$ & $86.44 \%(1.49 \sigma)$ \\
\cline { 2 - 5 } & $95.00 \%(1.96 \sigma)$ & $95.01 \%(1.96 \sigma)$ & $93.08 \%(1.82 \sigma)$ & $92.75 \%(1.80 \sigma)$ \\
\cline { 2 - 5 } & $99.00 \%(2.58 \sigma)$ & $99.00 \%(2.58 \sigma)$ & $98.51 \%(2.43 \sigma)$ & $98.42 \%(2.41 \sigma)$ \\
\cline { 2 - 5 } & $99.73 \%(3.00 \sigma)$ & $99.72 \%(2.99 \sigma)$ & $99.58 \%(2.86 \sigma)$ & $99.56 \%(2.85 \sigma)$ \\
\hline \multirow{4}{*}{ LMA } & $90.00 \%(1.64 \sigma)$ & $89.86 \%(1.64 \sigma)$ & $85.90 \%(1.47 \sigma)$ & $82.31 \%(1.35 \sigma)$ \\
\cline { 2 - 5 } & $95.00 \%(1.96 \sigma)$ & $94.93 \%(1.95 \sigma)$ & $92.35 \%(1.77 \sigma)$ & $90.57 \%(1.67 \sigma)$ \\
\cline { 2 - 5 } & $99.00 \%(2.58 \sigma)$ & $98.99 \%(2.57 \sigma)$ & $98.28 \%(2.38 \sigma)$ & $98.00 \%(2.33 \sigma)$ \\
\cline { 2 - 5 } & $99.73 \%(3.00 \sigma)$ & $99.73 \%(3.00 \sigma)$ & $99.52 \%(2.82 \sigma)$ & $99.45 \%(2.78 \sigma)$ \\
\hline \multirow{4}{*}{ LOW } & $90.00 \%(1.64 \sigma)$ & $92.53 \%(1.78 \sigma)$ & $86.59 \%(1.50 \sigma)$ & $83.70 \%(1.40 \sigma)$ \\
\cline { 2 - 5 } & $95.00 \%(1.96 \sigma)$ & $96.39 \%(2.10 \sigma)$ & $92.81 \%(1.80 \sigma)$ & $91.32 \%(1.71 \sigma)$ \\
\cline { 2 - 5 } & $99.00 \%(2.58 \sigma)$ & $99.33 \%(2.71 \sigma)$ & $98.34 \%(2.40 \sigma)$ & $97.98 \%(2.32 \sigma)$ \\
\cline { 2 - 5 } & $99.73 \%(3.00 \sigma)$ & $99.82 \%(3.12 \sigma)$ & $99.51 \%(2.81 \sigma)$ & $99.42 \%(2.76 \sigma)$ \\
\hline \multirow{4}{*}{ VO } & $90.00 \%(1.64 \sigma)$ & $86.29 \%(1.49 \sigma)$ & & $81.82 \%(1.34 \sigma)$ \\
\cline { 2 - 5 } & $95.00 \%(1.96 \sigma)$ & $92.99 \%(1.81 \sigma)$ & & $90.42 \%(1.67 \sigma)$ \\
\cline { 2 - 5 } & $99.00 \%(2.58 \sigma)$ & $98.68 \%(2.48 \sigma)$ & & $98.07 \%(2.34 \sigma)$ \\
\cline { 2 - 5 } & $99.73 \%(3.00 \sigma)$ & $99.69 \%(2.96 \sigma)$ & & $99.50 \%(2.81 \sigma)$ \\
\hline
\end{tabular}

TABLE II. Confidence level of the usual 90\%, 95\%, 99\% and 99.73\% CL allowed regions. The confidence levels have been calculated generating more than one million synthetic data sets. The first column indicates in which region the surrogate of the true values of the neutrino oscillation parameters has been assumed to be. The second column indicates the usual CL. The third column reports the confidence levels calculated locally, i.e. restricting the allowed values of the parameters around the region in which the assumed surrogates of the true values lie. The fourth column reports the confidence levels calculated restricting the allowed values of the parameters to the MSW region (9). The fifth column reports the confidence levels calculated without any restriction on the allowed values of the parameters.

and LOW regions ("MSW" column of Table III). Finally, we calculated the confidence levels without any restriction on the allowed values of the parameters, assuming the surrogates of the true values in the local minima of $X^{2}$ of the SMA, LMA, LOW and VO regions ("global" column of Table [1]).

One can see that the values of the confidence levels calculated locally for the SMA region, where the global minimum of $X^{2}$ lies, practically coincide with the standard ones ("standard CL" column of Table (11). However, when the allowed values of the parameters are extended to the whole MSW region (9) or to the MSW and VO regions (global), the confidence levels are significantly smaller than the standard ones.

The same trend, slightly more pronounced, is observed when the surrogates of the true values of the parameters are assumed to correspond to the local minima of $X^{2}$ in the LMA and LOW region, with even a small deviation of the local confidence levels from the standard ones (with unpredictable sign).

When the surrogates of the true values of the parameters are assumed to correspond 
to the local minimum of $X^{2}$ in the VO region (10), the confidence levels are significantly smaller than the standard ones, even those calculated locally. This is due to the fact that the linear approximation used in the calculation of the standard confidence levels is badly violated (there are several disjointed allowed VO regions with non-elliptical shapes).

Summarizing the results of this section, we have shown that the standard confidence levels of the allowed regions in the neutrino oscillation parameter space are approximately correct if only one of the SMA, LMA or LOW region is considered to be allowed a priori. If the oscillation parameters are restricted to the MSW region (9), the confidence levels are significantly smaller than the standard ones, with some uncertainty depending on the assumed surrogates of the true values of the parameters. If one does not impose any restriction on the values of the parameters, the confidence levels decrease further. If only the VO regions are considered to be allowed even the confidence levels calculated locally are significantly smaller than the standard ones.

\section{EXACT ALLOWED REGIONS}

In the previous section we have calculated the confidence level of the allowed regions in the neutrino oscillation parameter space obtained with the standard procedure based on Eq. (5). This calculation is approximate, because it is based on the assumption of a surrogate for the unknown true values of the neutrino oscillation parameters. Furthermore, we have seen that the value of the confidence level is different if the surrogate for the unknown true values of the neutrino oscillation parameters is assumed to be the value of the parameters in the global minimum of $X^{2}$ or in one of the local minima.

Luckily, there is a well-known procedure for constructing exact confidence intervals independently of the true values of the parameters. This procedure has been invented by Neyman in 1937 [39] (see also 40,34,41]). It guarantees that the resulting confidence intervals have correct frequentist coverage (see [42 46]), i.e. they belong to a set of confidence intervals obtained with different or similar, real or hypothetical experiments that cover the true values of the parameters with the desired probability given by the chosen confidence level. In this section we apply this method in order to find confidence intervals with proper coverage for the neutrino oscillation parameters.

Neyman's construction of exact frequentist confidence interval with $100 \beta \%$ confidence level starts with the choice of an appropriate estimator of the parameters under investigation. Then, for any possible value of the parameters one calculates an acceptance interval with probability $\beta$, i.e. an interval of the estimator that contains $100 \beta \%$ of the values of the estimator obtained in a large series of trials. Several methods are available for the construction of the acceptance intervals (see [40,34,41,43, 45, 46] and references therein). If the probability distribution of the estimator is known, the acceptance intervals can be calculated analytically; if not, one can calculate the acceptance intervals with numerical Monte Carlo methods. In general the acceptance intervals can be composed by disjoint sub-intervals. In the case of $n$ parameters the acceptance intervals are regions in the $n$-dimensional parameter space.

Once the $100 \beta \%$ acceptance interval for each possible value of the parameters is calculated, the $100 \beta \%$ confidence interval is simply composed by all the parameter values whose 
acceptance interval covers the measured value of the estimator (i.e. the actual estimate of the parameters). If the acceptance intervals are composed by disjoint sub-intervals, also the confidence interval is composed by disjoint sub-intervals. As we will see in the following, this is what happens in the case of solar neutrino oscillations.

Our implementation of Neyman's construction goes as follows. First we choose as estimator of neutrino oscillation parameters the values of the parameters in the minimum $X_{\min }^{2}$ of the least-squares function (11). Since the probability distribution of the chosen estimator is not known, we calculate it numerically with a Monte Carlo. We define an appropriate grid in the 2-dimensional space of the neutrino oscillation parameters $\tan ^{2} \vartheta, \Delta m^{2}$ and for each value of the parameters on the grid we generate a large number of synthetic data sets. For each data set we find the value of the parameters corresponding to the minimum of $X^{2}$. This procedure gives the distribution of $X_{\min }^{2}$ for each value of the parameters on the grid. Unfortunately this is a rather lengthy task that requires several days of computer time in order to reach an acceptable accuracy, essentially because of the large number of points on a reasonably fine grid, about five thousand in the MSW region (9) and six thousand in the $\mathrm{VO}$ region (10).

We define the $100 \beta \%$ acceptance intervals in the simplest and most natural way?: for each value of the parameters we choose the shortest possible acceptance interval, i.e. that containing the values of the parameters on the grid with highest probability, whose sum is equal or larger than $\beta$ (in general perfect equality is not reached because of the discrete nature of the grid). In the case of a linear least-squares fit this method gives the allowed regions obtained with the standard prescription (5). Therefore, our exact allowed regions can be compared directly with the standard ones.

The acceptance intervals are 2-dimensional regions in the $\tan ^{2} \vartheta-\Delta m^{2}$ parameter space. Because of the non-linearity of the neutrino oscillation probability as a function of the parameters, the acceptance intervals are composed by disjoint sub-intervals. This generates 2-dimensional confidence intervals composed by disjoint sub-intervals, some of which far from the values of the parameters corresponding to the actual $X_{\min }^{2}$. The confidence intervals are composed by the values of the parameters whose acceptance interval includes the parameters corresponding to the actual $X_{\min }^{2}$ (the measured value of the estimator).

The procedure is illustrated qualitatively in Fig. 1, where the cross corresponds to the actually measured $X_{\min }^{2}$, the union of the two vertically hatched regions is the acceptance interval associated with $\tan ^{2} \vartheta_{A}, \Delta m_{A}^{2}$, and the union of the three horizontally hatched regions is the acceptance interval associated with $\tan ^{2} \vartheta_{B}, \Delta m_{B}^{2}$. Since the acceptance interval associated with $\tan ^{2} \vartheta_{A}, \Delta m_{A}^{2}$ includes the point corresponding to $X_{\min }^{2}$, the point $\tan ^{2} \vartheta_{A}, \Delta m_{A}^{2}$ belongs to the confidence interval. On the other hand, the acceptance interval associated with $\tan ^{2} \vartheta_{B}, \Delta m_{B}^{2}$ does not include the point corresponding to $X_{\text {min }}^{2}$ and the point $\tan ^{2} \vartheta_{A}, \Delta m_{A}^{2}$ is out of the confidence interval.

5 There is a subtle problem in choosing the method that defines the acceptance intervals: the method must be chosen independently of the data and the result. This is what we have done. Otherwise, the property of coverage is lost (see 42, 41, 43, 46]), and one can always choose a method "ad hoc" to obtain any desired result. 


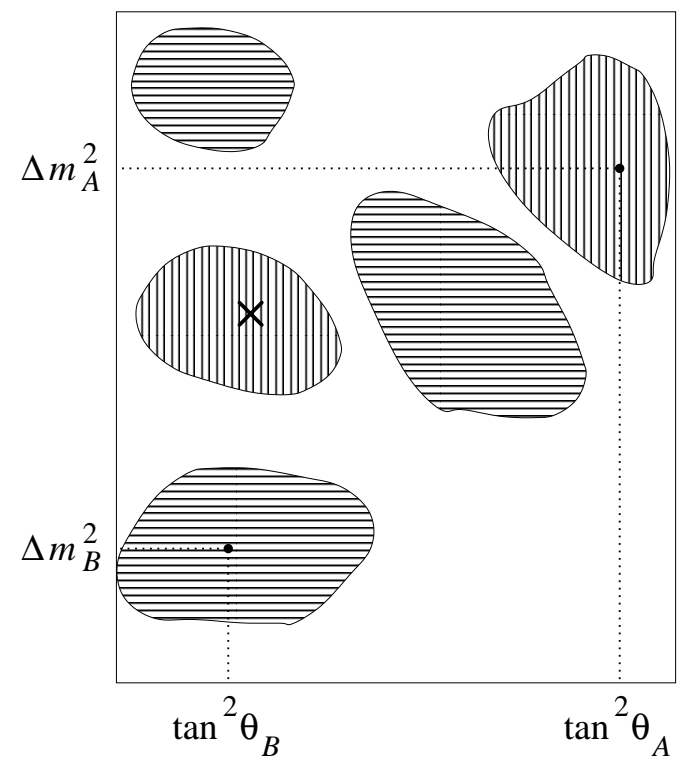

FIG. 1. Illustration of the acceptance intervals. The cross corresponds to the actual $X_{\min }^{2}$. The two vertically hatched regions constitute the acceptance interval associated with $\tan ^{2} \vartheta_{A}, \Delta m_{A}^{2}$. The three horizontally hatched regions constitute the acceptance interval associated with $\tan ^{2} \vartheta_{B}$, $\Delta m_{B}^{2}$.

The results of our calculations are presented in Figs. 2 25, where we have depicted the 90\%, 95\%, 99\% and 99.73\% CL regions (gray areas) confronted with those obtained with the standard method based on Eq. (5) (areas enclosed by solid lines).

In Fig. 2 we have restricted the possible values of the neutrino oscillation parameters in a region around the SMA solution, where $X_{\min }^{2}$ lies. The acceptance interval for each point on the grid in the parameter space has been calculated generating about $6 \times 10^{5}$ synthetic data sets (different for different points on the grid, in order to avoid correlations). One can see that the standard allowed SMA region is an acceptable approximation of the exact ${ }^{\circ}$ confidence interval. This is due to the fact that locally the linear approximation is rather good, as we already found in the previous two sections.

In Fig. 3 we have extended the possible values of the neutrino oscillation parameters to all the MSW region (9). For this figure the number of synthetic data sets for each point on the grid is about $7 \times 10^{4}$ (less than in Fig. 2 because of the larger size of the grid, that slows down the calculation). The standard SMA region is still an acceptable approximation of the exact SMA region, but the exact LMA and LOW regions are dramatically larger than the

${ }^{6}$ Here the adjective "exact" refers to the method, that produces confidence intervals with exact coverage. Obviously our confidence intervals are approximations of the exact ones, that would be obtained with an infinitely dense grid in parameter space and an infinite set of synthetic random data sets. 
standard ones, so large that they merge together, producing a huge allowed region around maximal mixing $\left(\tan ^{2} \vartheta=1\right)$. This is true even at $90 \% \mathrm{CL}$.

Figures 4 and 5 show, respectively, the allowed MSW and VO regions when there is no restriction on the possible values of the neutrino oscillation parameters (the number of synthetic data sets for each point on the grid is now about $6.5 \times 10^{4}$ ). Again, one can see that the standard SMA region is an acceptable approximation of the exact SMA region, but the exact LMA, LOW and VO regions are much larger than the standard ones.

From the results of our calculations we conclude that the standard method to calculate allowed regions produces reliable results only locally, i.e. in the calculation of the allowed region surrounding the global minimum of $X^{2}$. The other allowed regions are dramatically underestimated by the standard method.

\section{CONCLUSIONS}

We have presented the results of a numerical Monte Carlo calculation of the goodness of fit and the confidence level of the standard allowed regions for the neutrino oscillation parameters $\Delta m^{2}, \tan ^{2} \vartheta$ obtained from the fit of solar neutrino data. We have shown that the standard values of the goodness of fit and of the confidence level of the allowed regions are significantly overestimated with the standard method. This is due to the non-linear dependence of the neutrino oscillation probability from the parameters. The linear approximation, leading to the standard values of the goodness of fit and of the confidence level of the allowed regions, is valid only locally, for values of the parameters around a specific MSW solution (SMA, LMA, LOW). In the case of the VO solution the linear approximation is not valid even locally, because of the strong non-linearity of the oscillation probability that causes the existence of several allowed regions close together.

We have also calculated exact allowed regions with correct frequentist coverage using Neyman's method. The results of these calculations show that the standard allowed region around the global minimum of the least-squares function is a reasonable approximation of the exact one. On the other hand, the size of the other regions is dramatically underestimated in the standard method. Indeed, in our calculation the exact SMA region, that contains the minimum of the least-squares function, practically coincides with the standard one. On the other hand, the exact LMA and LOW regions are much larger than the standard ones, so much that they merge in a huge allowed region around maximal mixing. Also the exact allowed VO regions are much larger than the standard ones.

The indications on neutrino mixing coming from solar neutrino data are becoming increasingly important for theory and experiment. Furthermore, solar neutrino data will soon be enriched by results of new powerful experiments (SNO [47, Borexino [48, GNO 49] and others [50]). As we have shown, the standard statistical analysis of solar neutrino data can lead to incorrect conclusions concerning the goodness of fit, the confidence level of the allowed regions and the size of the allowed regions far from the global minimum of the least-squares function. Hence, we believe that it is time to examine critically the method of

statistical analysis of solar neutrino data and bring it to the level of quality already attained in other branches of research in high-energy physics. 

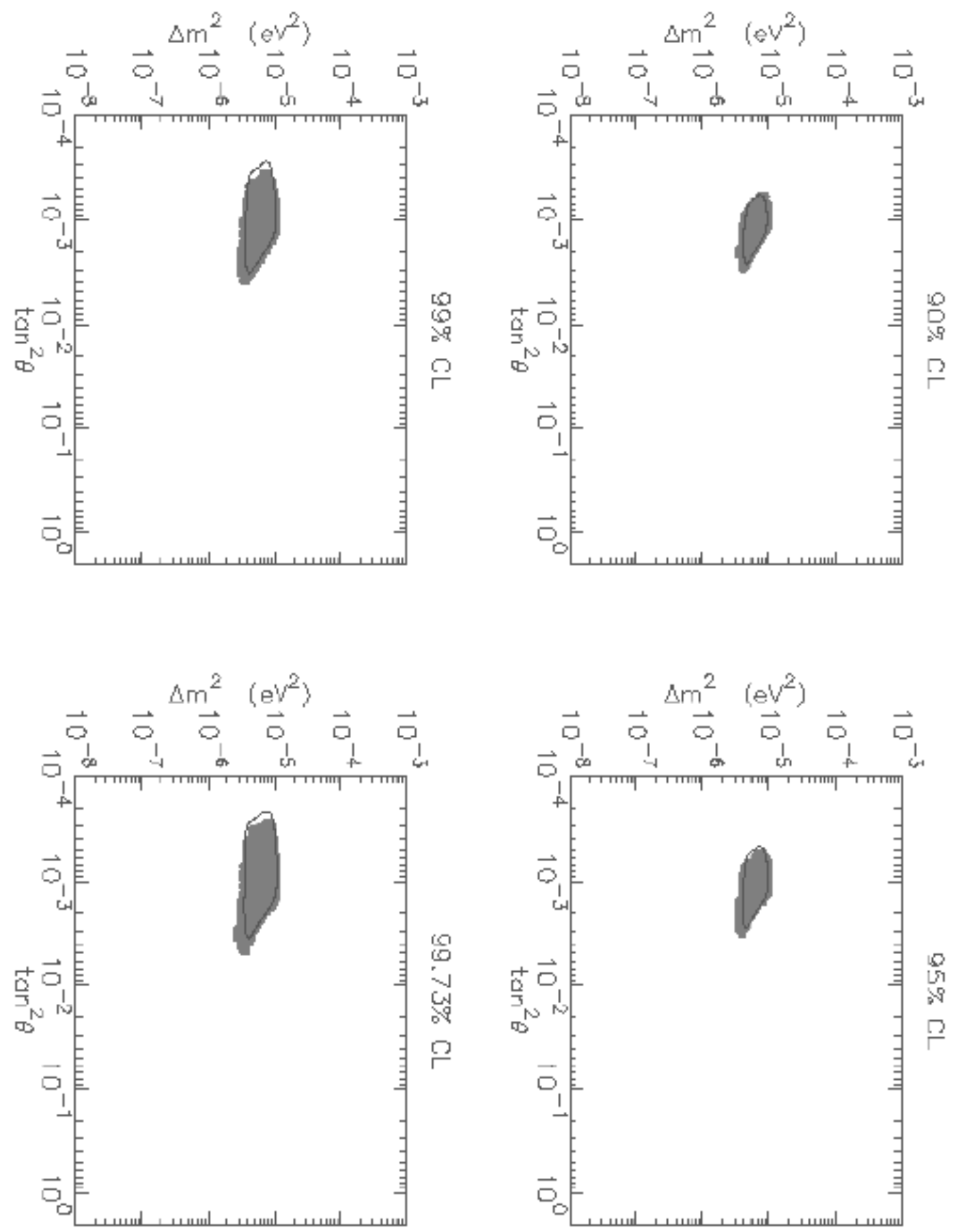

FIG. 2. Allowed 90\%, 95\%, 99\%, 99.73\% confidence level regions in the $\tan ^{2} \vartheta-\Delta m^{2}$ plane. In each plot the gray area is the allowed region with exact frequentist coverage obtained restricting the possible values of $\tan ^{2} \vartheta$ and $\Delta m^{2}$ in a region around the SMA solution, where $X_{\min }^{2}$ lies. The area enclosed by the solid line is the standard SMA allowed region. 

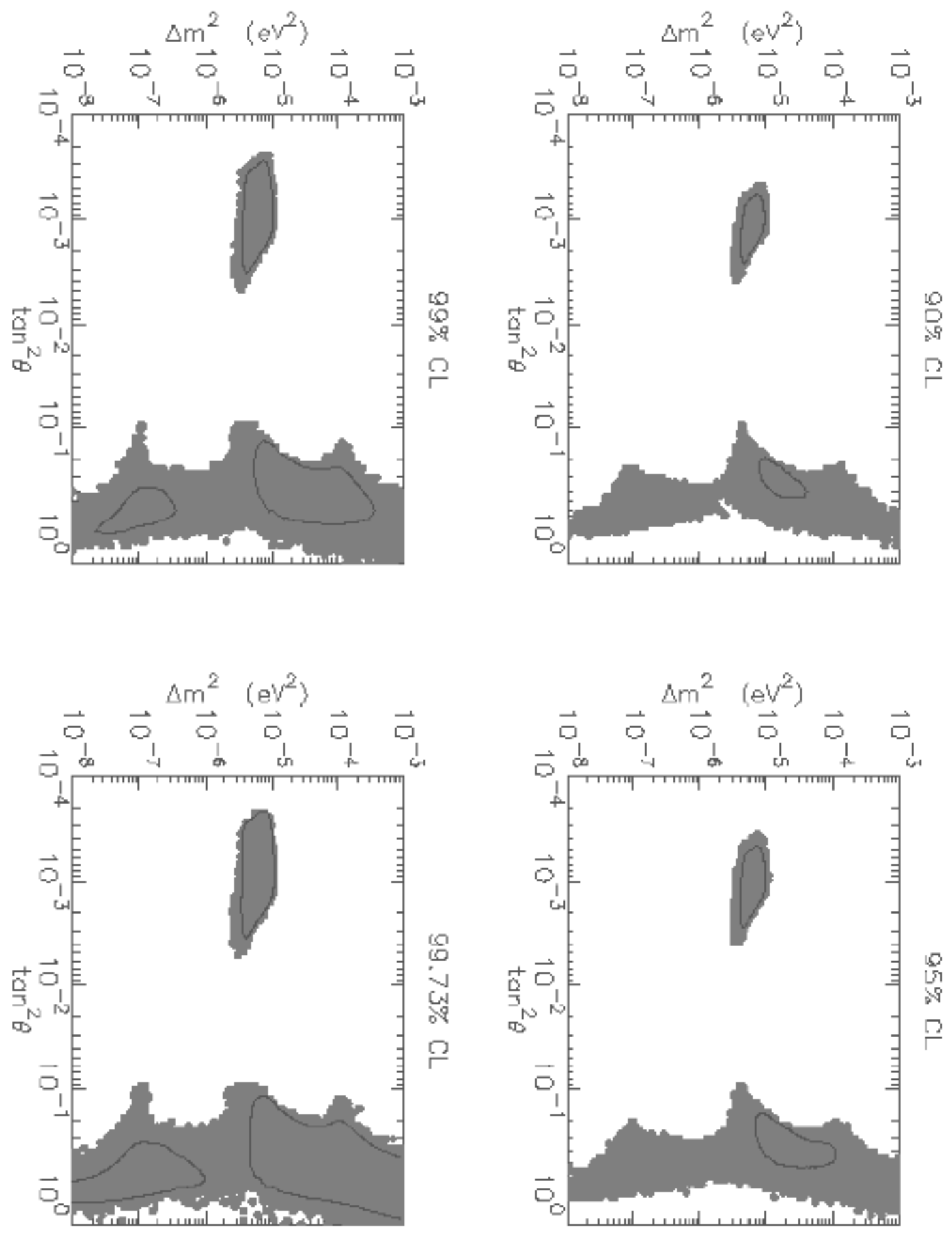

FIG. 3. Allowed 90\%, 95\%, 99\%, 99.73\% confidence level regions in the $\tan ^{2} \vartheta-\Delta m^{2}$ plane. The gray areas are the allowed regions with exact frequentist coverage obtained considering all possible values of $\tan ^{2} \vartheta$ and $\Delta m^{2}$ in the MSW region (the whole area of the plots). The areas enclosed by the solid lines are the standard SMA, LMA and LOW allowed region. 

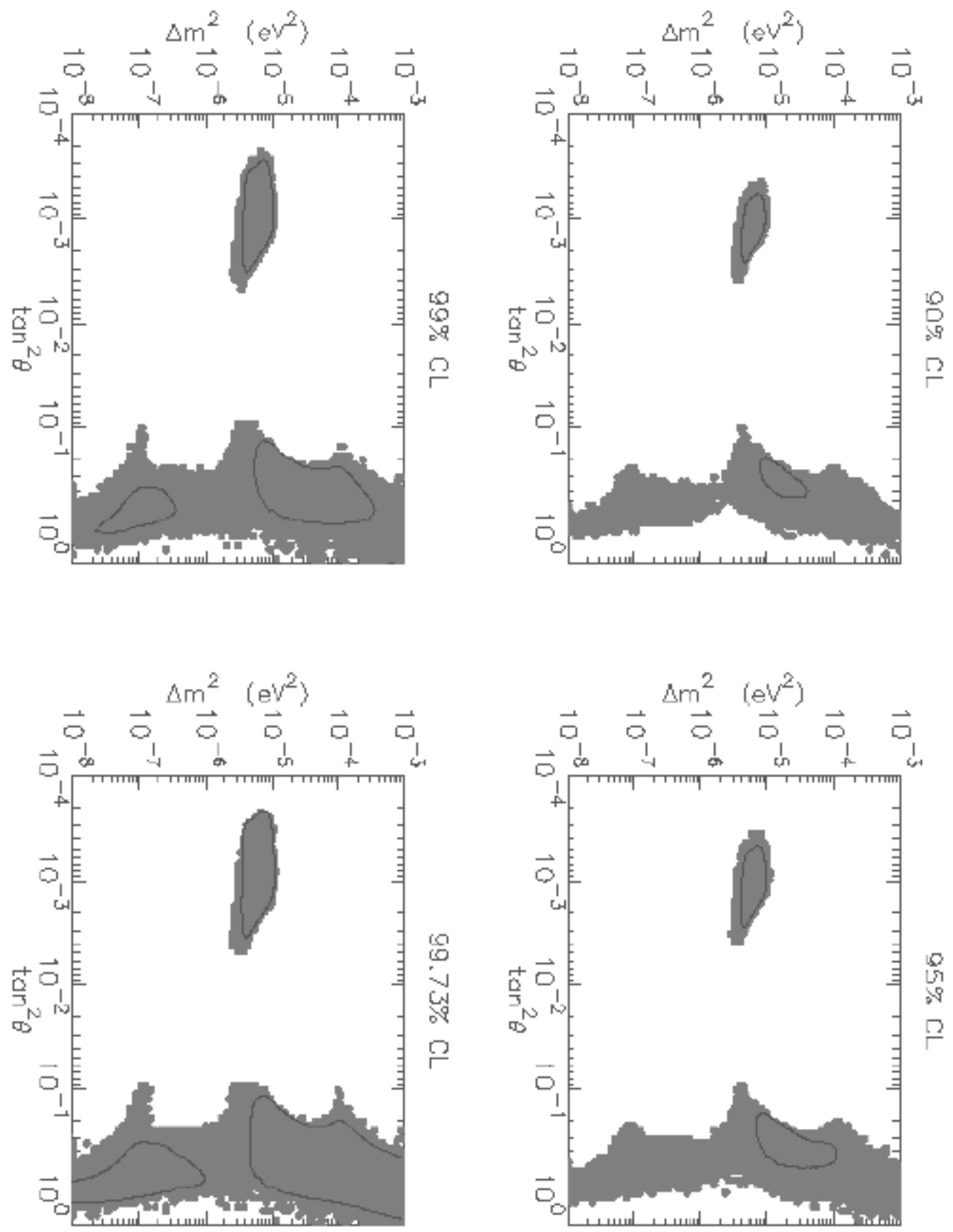

FIG. 4. Allowed 90\%, 95\%, 99\%, 99.73\% confidence level regions in the $\tan ^{2} \vartheta-\Delta m^{2}$ plane. The gray areas are the allowed regions with exact frequentist coverage in the MSW region obtained without any restriction on the possible values of $\tan ^{2} \vartheta$ and $\Delta m^{2}$. The areas enclosed by the solid lines are the standard SMA, LMA and LOW allowed region. 

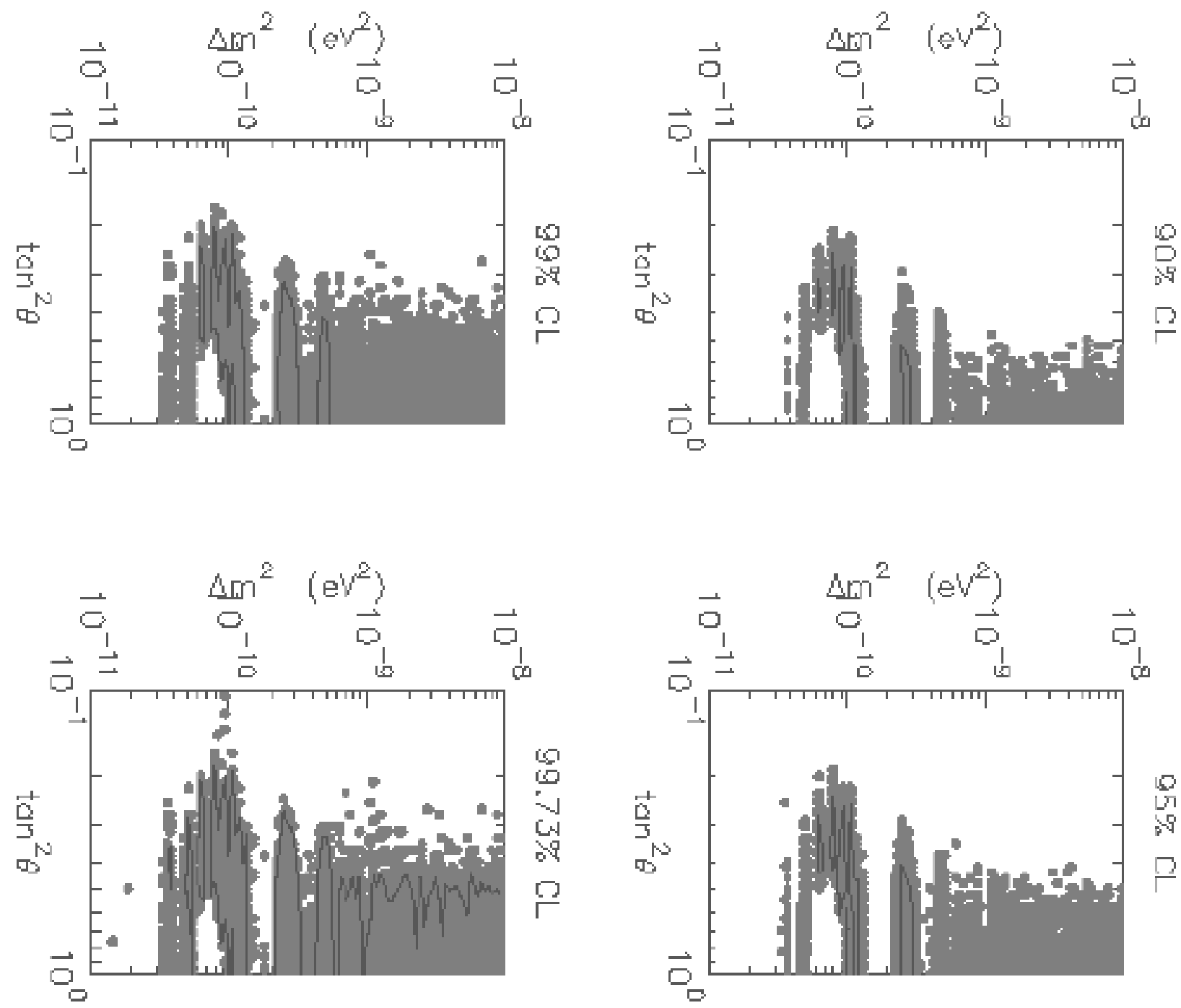

FIG. 5. Allowed 90\%, 95\%, 99\%, 99.73\% confidence level regions in the $\tan ^{2} \vartheta-\Delta m^{2}$ plane. The gray areas are the allowed regions with exact frequentist coverage in the VO region obtained without any restriction on the possible values of $\tan ^{2} \vartheta$ and $\Delta m^{2}$. The areas enclosed by the solid lines are the standard VO allowed regions. 


\section{REFERENCES}

[1] B. T. Cleveland et al., Astrophys. J. 496, 505 (1998).

[2] Y. Suzuki (Super-Kamiokande Coll.), Talk presented at the XIX Int. Symposium on Lepton and Photon Interactions at High Energies, Stanford, Aug. 1999 (http://wwwsk.icrr.u-tokyo.ac.jp/doc/sk/pub/index.html).

[3] GALLEX, W. Hampel et al., Phys. Lett. B447, 127 (1999).

[4] SAGE, J. N. Abdurashitov et al., Phys. Rev. C60, 055801 (1999), arXiv:astro$\mathrm{ph} / 9907113$.

[5] M.C. Gonzalez-Garcia, P.C. de Holanda, C. Pena-Garay and J.W.F. Valle, arXiv:hep$\mathrm{ph} / 9906469$.

[6] Neutrino 2000, Sudbury, Canada, June 2000 (http://nu2000.sno.laurentian.ca).

[7] M.V. Garzelli and C. Giunti, work in progress.

[8] S. M. Bilenky and S. T. Petcov, Rev. Mod. Phys. 59, 671 (1987).

[9] S. M. Bilenky, C. Giunti, and W. Grimus, Prog. Part. Nucl. Phys. 43, 1 (1999), arXiv:hep-ph/9812360.

[10] A. de Gouvea, A. Friedland and H. Murayama, arXiv:hep-ph/0002064.

[11] M.C. Gonzalez-Garcia and C. Pena-Garay, arXiv:hep-ph/0002186.

[12] G. L. Fogli, E. Lisi, and G. Scioscia, Phys. Rev. D52, 5334 (1995), hep-ph/9506350.

[13] G. L. Fogli, E. Lisi, and D. Montanino, Phys. Rev. D54, 2048 (1996), arXiv:hep$\mathrm{ph} / 9605273$.

[14] G. L. Fogli, E. Lisi, D. Montanino, and G. Scioscia, Phys. Rev. D56, 4365 (1997), hep-ph/9706230.

[15] C. Giunti, M. C. Gonzalez-Garcia, and C. Pena-Garay, Phys. Rev. D62, 013005 (2000), arXiv:hep-ph/0001101.

[16] S.P. Mikheyev and A.Yu. Smirnov, Yad. Fiz. 42, 1441 (1985) [Sov. J. Nucl. Phys. 42, 913 (1985)]; Il Nuovo Cim. C 9, 17 (1986); L. Wolfenstein, Phys. Rev. D 17, 2369 (1978); Phys. Rev. D 20, 2634 (1979).

[17] T. K. Kuo and J. Pantaleone, Rev. Mod. Phys. 61, 937 (1989).

[18] P. I. Krastev and S. T. Petcov, Phys. Lett. B207, 64 (1988).

[19] P. I. Krastev and S. T. Petcov, Phys. Lett. B299, 99 (1993).

[20] P. I. Krastev and S. T. Petcov, Phys. Lett. B285, 85 (1992).

[21] S. J. Parke, Phys. Rev. Lett. 57, 1275 (1986).

[22] S. T. Petcov, Phys. Lett. B200, 373 (1988).

[23] Q. Y. Liu, M. Maris, and S. T. Petcov, Phys. Rev. D56, 5991 (1997), arXiv:hep$\mathrm{ph} / 9702361$.

[24] S. T. Petcov, Phys. Lett. B434, 321 (1998), arXiv:hep-ph/9805262.

[25] E. K. Akhmedov, Nucl. Phys. B538, 25 (1999), arXiv:hep-ph/9805272.

[26] M. V. Chizhov and S. T. Petcov, Phys. Rev. Lett. 83, 1096 (1999), hep-ph/9903399.

[27] M.V. Chizhov and S.T. Petcov, arXiv:hep-ph/9903424.

[28] J.N. Bahcall, WWW page: http://www.sns.ias.edu/ jnb/.

[29] A. Friedland, arXiv:hep-ph/0002063.

[30] G.L. Fogli, E. Lisi, D. Montanino and A. Palazzo, arXiv:hep-ph/0005261.

[31] G. L. Fogli and E. Lisi, Astropart. Phys. 3, 185 (1995). 
[32] G. L. Fogli, E. Lisi, D. Montanino, and A. Palazzo, Phys. Rev. D62, 013002 (2000), arXiv:hep-ph/9912231.

[33] M.V. Garzelli and C. Giunti, arXiv:hep-ph/0006026.

[34] W.T. Eadie, D. Drijard, F.E. James, M. Roos and B. Sadoulet, Statistical Methods in Experimental Physics, North Holland, Amsterdam, 1971.

[35] A.G. Frodesen, O. Skjeggestad and H. Tofte, Probability and statistics in particle physics, Universitetsforlaget, Bergen, Norway, 1979.

[36] W.H. Press, S.A. Teukolsky, W.T. Vetterling and B.P. Flannery, Numerical Recipes in C, Cambridge University Press, Second Edition, 1992.

[37] J. N. Bahcall, P. I. Krastev, and A. Y. Smirnov, Phys. Rev. D58, 096016 (1998), arXiv:hep-ph/9807216.

[38] A. Stuart and J.K. Ord, Kendall's Advanced Theory of Statistics, Vol. 1, Distribution Theory Sixth Edition, Halsted Press, 1994.

[39] J. Neyman, Philos. Trans. R. Soc. London Sect. A 236, 333 (1937), reprinted in A selection of Early Statistical Papers on J. Neyman, University of California, Berkeley, 1967, p. 250.

[40] A. Stuart, J.K. Ord and S. Arnold, Kendall's Advanced Theory of Statistics, Vol. 2A, Classical inference and the linear model Sixth Edition, Oxford University Press, 1999.

[41] C. Caso et al., Eur. Phys. J. C3, 1 (1998).

[42] R.D. Cousins, Am. J. Phys. 63, 398 (1995).

[43] G.J. Feldman and R.D. Cousins, Phys. Rev. D 57, 3873 (1998), arXiv:physics/9711021.

[44] C. Giunti, Phys. Rev. D59, 113009 (1999), arXiv:hep-ex/9901015.

[45] C. Giunti and M. Laveder, arXiv:hep-ex/0002020.

[46] C. Giunti, hep-ex/0002042, Talk presented at the Workshop on "Confidence Limits", CERN, 17-18 January 2000.

[47] J. Boger et al., arXiv:nucl-ex/9910016.

[48] Borexino, F. von Feilitzsch, Prog. Part. Nucl. Phys. 40, 123 (1998).

[49] GALLEX and GNO, T. A. Kirsten, Nucl. Phys. Proc. Suppl. 77, 26 (1999).

[50] F. von Feilitzsch, Talk presented at Neutrino 2000, Sudbury, Canada, June 2000 (http://nu2000.sno.laurentian.ca). 